\title{
Energy Metabolism Analysis of Three Different Mesenchymal Stem Cell Populations of Umbilical Cord Under Normal and Pathologic Conditions
}

\author{
Eleonora Russo ${ }^{1,2} \cdot$ Jea-Young Lee ${ }^{1} \cdot$ Hung Nguyen ${ }^{1} \cdot$ Simona Corrao $^{2} \cdot$ Rita Anzalone $^{3} \cdot$ Giampiero La Rocca $^{2}$. \\ Cesar V. Borlongan ${ }^{1}$
}

Published online: 17 March 2020

(C) The Author(s) 2020

\begin{abstract}
Human umbilical cord mesenchymal stem cells (hUC-MSCs) are a pivotal source of therapeutically active cells for regenerative medicine due to their multipotent differentiation potential, immunomodulatory and anti-inflammatory proprieties, as well as logistical collection advantages without ethical concerns. However, it remains poorly understood whether MSCs from different compartments of the human umbilical cord are therapeutically superior than others. In this study, MSCs were isolated from Wharton's jelly (WJ-MSCs), perivascular region (PV-MSCs) and cord lining (CL-MSCs) of hUC. These cells expressed the mesenchymal markers (CD90, CD73), stemness marker (OCT4), endothelial cell adhesion molecular marker (CD146), and the monocyte/macrophage marker (CD14) found within the MSC population implicated as a key regulator of inflammatory responses to hypoxia, was displayed by WJ-, PV-, and CL-MSCs respectively. A direct consequence of oxygen and glucose deprivation during stroke and reperfusion is impaired mitochondrial function that contributes to cellular death. Emerging findings of mitochondria transfer provide the basis for the replenishment of healthy mitochondria as a strategy for the treatment of stroke. Cell Energy Phenotype and Mito Stress tests were performed the energy metabolic profile of the three MSC populations and their mitochondrial function in both ambient and OGD cell culture conditions. PV-MSCs showed the highest mitochondrial activity. CL-MSCs were the least affected by OGD/R condition, suggesting their robust survival in ischemic environment. In this study, MSC populations in UC possess comparable metabolic capacities and good survival under normal and hypoxic conditions suggesting their potential as transplantable cells for mitochondrial-based stem cell therapy in stroke and other ischemic diseases.
\end{abstract}

Keywords Umbilical cord mesenchymal stem cells $\cdot$ Wharton's Jelly $\cdot$ Perivascular $\cdot$ Stroke $\cdot$ Ischemic diseases $\cdot$ Mitochondria . Bioenergetics $\cdot$ Stem cell therapy

\section{Introduction}

Stroke is the second leading cause of death and disability worldwide behind heart diseases [1]. A direct consequence

Giampiero La Rocca

giampylr@hotmail.com

$\triangle$ Cesar V. Borlongan

cborlong@health.usf.edu

1 Department of Neurosurgery and Brain Repair, University of South Florida Morsani College of Medicine, Tampa, Florida, USA

2 Section of Histology and Embryology, Department of Biomedicine, Neurosciences and Advanced Diagnostics (BIND), University of Palermo, Palermo, Italy

3 Department of Surgical, Oncological and Stomatological Sciences, University of Palermo, Palermo, Italy of oxygen and glucose deprivation (OGD) during stroke is the dysfunction of mitochondria that impairs oxidative metabolism and contributes to oxidative stress, neuronal death and inflammation [2]. Indeed, mitochondria are responsible for more than $90 \%$ of the total adenosine triphosphate (ATP) demand of the cell [3]. Accordingly, the decrease of ATP production following OGD leads to energy failure, excitotoxicity and calcium overload that, in turn, determine loss of mitochondrial membrane potential [2]. Damaged mitochondria are characterized by an increase of membrane permeability that allows the release of pro-apoptotic molecules in the cytoplasm triggering apoptotic cell death [2]. Thus, mitochondrial dysfunction plays a central role in stroke injury.

Cell-based therapies aim to replace dead cells and promote the survival of damaged cells, altogether directly aiding exogenous and endogenous repair mechanisms or indirectly, by providing trophic support and reducing inflammatory 
response [4, 5]. Recently, a novel therapeutic mechanism of stem cells has been demonstrated to involve the transfer of healthy mitochondria into damaged cells [6]. Mitochondria can be released through tunneling nanotubes (TNTs), microvesicles, gap junctions, cell fusion and direct uptake of isolated mitochondria [7]. Even though the signals that induce a cell to release its own mitochondria and transfer these organelles to another cell are not still clear, multiple converging lines of evidence suggests that this phenomenon can help damaged cells to recover their functions [8]. In the last few years, mitochondrial transfer has been shown to occur between several cell types, including mesenchymal stem cells (MSCs), astrocytes and neurons, and endothelial progenitor cells $[9,10]$. Taken together, these observations suggest that the transfer of healthy mitochondria into damaged cells may be a novel therapeutic strategy for stroke.

Human umbilical cord (hUC)-derived MSCs (hUC-MSCs) are an enticing cellular source for regenerative medicine purposes due to their self-renewal and multipotent differentiation potential as well as to their immunomodulatory and antiinflammatory abilities $[5,11,12]$. MSCs have been isolated from Wharton's jelly (WJ), perivascular region (PV) and cord lining (CL) of hUC [13]. However, it is still unclear whether MSCs from a certain compartment of hUC are therapeutically superior to MSCs from other compartments [13]. Interestingly, because hUC is composed only of two arteries and a vein [13], the hUC-MSCs are physiologically adapted to survive in a relatively hypoxic and glucose-poor environment leading to the overarching hypothesis that these cells may have a therapeutic potential for the treatment of ischemic pathologies, such as stroke.

The aim of the current study was to analyze the live-cell metabolic profile and mitochondrial function of all the three hUC-MSC populations in both normal and pathological stroke conditions in vitro.

\section{Materials and Methods}

\section{Isolation and Culture of MSCs from Different Regions of Umbilical Cord}

Human umbilical cords $(n=03)$ were purchased from ZenBio and they were obtained after mothers' informed consent, immediately after full-term births with normal vaginal delivery. The isolation of MSCs from perivascular, WJ and cord lining regions of the hUC was performed as previously described [14-16]. In particular, an enzymatic method was chosen for the isolation of PV-MSCs in order to increase the cellular harvested yield around the vessels, according to Sarugaser et al. [15]. On the other hand, an explant method was performed for the isolation of both WJ- and CL-MSCs, according to Kita et al. and Mennan et al. [14, 16]. Although the isolation methods differed, the chosen technique for harvesting MSCs from each region led to optimal cell survival and amplification across the three hUC-MSC sources. For all the three cellular types, the culture medium was consisted of DMEM low-glucose (Sigma), supplemented with 10\% FBS (Gibco), 1x NEAA (Sigma) and 1x antibiotics-antimycotics (Gibco) and replaced every 2-3 days until the cells were ready for sub-culture (complete medium).

Briefly, the hUCs were washed in 1x phosphate buffered saline (PBS) in order to remove bloodstains and then rinsed in warm HBSS (Gibco) supplemented by $2 \mathrm{x}$ antibiotics/ antimycotics (Gibco). Subsequently, the hUC was cut into pieces of about 5-6 cm length and then carefully sectioned longitudinally to expose the $\mathrm{WJ}$ and the blood vessels.

For the isolation of PV-MSCs, the three vessels were isolated using forceps and scalpel and placed in $40 \mathrm{ml}$ of HBSS (Gibco) supplemented with $100 \mathrm{U} / \mathrm{mL}$ Type I Collagenase (Sigma) and $0.01 \mathrm{U} / \mathrm{mL}$ Hyaluronidase (Stemcell Technologies) in a $50 \mathrm{~mL}$ Falcon tube and left to digest in for $4 \mathrm{~h}$ at $37^{\circ} \mathrm{C}$. After the digestion was completed, all the vessels were removed from the suspension using forceps. The suspension, containing the cells, was centrifuged at $285 \mathrm{~g}$ for $10 \mathrm{~min}$. Subsequently, the supernatant was discarded and the cellular pellet was treated with $50 \mathrm{ml}$ of $0.8 \%$ ammonium chloride (Stemcell Technologies) and incubated at room temperature for $5 \mathrm{~min}$ to lyse the erythrocytes. Thereafter, the tube was centrifuged for $10 \mathrm{~min}$ at $285 \mathrm{~g}$ and the supernatant was discarded. The cells, obtained from the perivascular region of each hUC, were counted and plated in one non-coated T-75 tissue culture flask with complete medium and put it in 5\% $\mathrm{CO}_{2}$ incubator at $37^{\circ} \mathrm{C}$.

For isolation of WJ-MSCs and CL-MSCs, after removing the vessels, the remaining cord was cut in smaller pieces with a length of about $1.5 \mathrm{~cm}$ and each piece was placed in a well of a non-coated 6-well culture plate with complete medium and incubated overnight in $5 \% \mathrm{CO}_{2}$ at $37^{\circ} \mathrm{C}$. The following day, the $\mathrm{WJ}$ absorbed the complete medium containing phenol red and, thus, it can be distinguished from the CL that is about $1 \mathrm{~mm}$ width. The WJ was separated from the CL from each piece of hUC by using a scalpel. The collected $\mathrm{WJ}$ was plated in a well of a non-coated 6-well culture plate and each piece of the remaining CL was plated in a well of another non-coated 6-well plate with the sub-amnion side touching the bottom of the well to allow the sub-amnion cellular exit and attachment to the culture plate. The $\mathrm{WJ}$ and $\mathrm{CL}$ were cultured with complete medium changed every 2-3 days over for 15 days in culture, and the cellular exit from both tissues, as well as the attachment to the plastic surface of the tissue culture plate, was monitored by phase-contrast microscopy. After 15 days, the $\mathrm{WJ}$ and $\mathrm{CL}$ tissues were removed and the attached cells were cultured until reaching the confluence.

The isolated cells were subcultured until passage 4 for all the three cellular types. Morphological analysis was 
performed by observing the cells at the phase contrast microscope Olympus. For all subsequent experiments, a minimum of three independent replicates was done.

\section{Immunocytochemistry/ IF}

Cells were seeded at the density of $1 \times 10^{4}$ cells $/ \mathrm{cm}^{2}$ in 8 -well chamber slides and cultured for 2 days. Subsequently the cells were fixed with $4 \%$ paraformaldehyde in PBS for $20 \mathrm{~min}$ at room temperature. After a wash with PBS, $0.1 \%$ Triton X-100 $(\mathrm{w} / \mathrm{v})$ in PBS was added and kept incubated for $5 \mathrm{~min}$ to allow the permeabilization. After rinsing with PBS, nonspecific binding was blocked by incubating 3\% normal goat serum (Invitrogen 50-062Z) in $0.1 \%$ Triton-X100 in PBS for $1 \mathrm{~h}$ at room temperature. Each primary antibody $(\mathrm{Ab})$ was diluted in blocking buffer and incubated with samples overnight at $4{ }^{\circ} \mathrm{C}$. Dilution of each primary $\mathrm{Ab}$ is specified in Table 1. After 3 washes (5 min for each wash) with PBS, secondary Abs were added for $1 \mathrm{~h}$ at room temperature (Table 1). Next, additional 3 washes were performed, each 5 min with PBS, then the slides were mounted with Antifade Mounting Medium with DAPI (Vectashield HardSet H-1500) and fluorescent images were collected using a Zeiss microscope. Three independent observers evaluated the immunohistochemical results and quantified the percentage of positive cells for each marker.

\section{Oxygen-Glucose Deprivation/Reperfusion}

PV-, WJ- and CL-MSCs were seeded at the density of 10,000 cells $/ \mathrm{cm}^{2}$ and cultured until reaching confluence. The cells were exposed to OGD/R to mimic the ischemic and the reperfusion condition following stroke as previously described [17]. Briefly, after the removal of the growth medium, the cells were exposed to PBS and placed in an anaerobic chamber (Plas Labs) containing nitrogen $\left(95 \% \mathrm{~N}_{2}\right)$ and carbon dioxide $\left(5 \% \mathrm{CO}_{2}\right)$ for $15 \mathrm{~min}$ at $37^{\circ} \mathrm{C}$. Finally, the chamber was sealed and incubated for $90 \mathrm{~min}$ at $37^{\circ} \mathrm{C}$ (hypoxic-ischemic condition). OGD was terminated by removal of PBS, and addition of the growth medium, then the cell cultures were then reintroduced to the regular $95 \% \mathrm{O}_{2}$ and $5 \% \mathrm{CO}_{2}$ incubator (normoxic condition) at $37^{\circ} \mathrm{C}$ for $24 \mathrm{~h}$, which represented a model of "reperfusion."

Table 1 Antibodies used for the immunofluorescence staining

\section{Measurement of Cell Viability}

Measurement of cell viability was performed using fluorescent live cell assay. Both control and OGD/R treated cells were incubated with $1 \mu \mathrm{M}$ Calcein-AM (Trevigen) for $30 \mathrm{~min}$ in the regular $95 \% \mathrm{O}_{2}$ and $5 \% \mathrm{CO}_{2}$ incubator at 37 ${ }^{\circ} \mathrm{C}$. The green fluorescence of the live cells was measured by the EnSpire Multimode Plate Reader $(E x / E m=490 / 520$; Perkin Elmer). In addition, a morphological analysis was performed using a phase contrast microscope (Olympus).

\section{Cell Energy Phenotype Test}

PV-, WJ- and CL-MSCs were seeded into the Seahorse XF96 Cell Culture Microplates (Agilent) at the cell density of $10,000 \mathrm{cell} / \mathrm{s} / \mathrm{cm}^{2}$ and cultured until confluence. Prior to the start of the Seahorse XF Cell Energy Phenotype test, a sensor cartridge was hydrated in Seahorse XF Calibrant (Agilent) following the manufacturer's instruction (Agilent). On the day of the assay, the cells were washed once and incubated in XF Seahorse Base Medium DMEM (Agilent) supplemented with $10 \mathrm{mM}$ glucose, $1 \mathrm{mM}$ sodium pyruvate, and $2 \mathrm{mM} \mathrm{L-}$ glutamine. The XF Seahorse Base Medium DMEM was prepared following the manufacturer's instruction (Agilent), but with slight modification. Although the Agilent protocol suggests two washes, the cells were rinsed only one time to avoid the risk of detaching the cells from the plate. After calibration of the Seahorse XF96 Analyzer (Agilent), the sensor cartridge was removed from the instrument and Seahorse XF96 Cell Culture Microplate was inserted. For all the three types of cells, the oxygen consumption rate (OCR) and the extracellular acidification rate (ECAR) readings were taken over time under basal conditions and after the addition of mitochondrial inhibitors Oligomycin $(1 \mu \mathrm{M})$ and carbonilcyanide $\mathrm{p}$ triflouromethoxyphenylhydrazone (FCCP, $1 \mu \mathrm{M})$. With the simultaneous injection of these stressor compounds two events occurred: Oligomycin inhibited ATP production by the mitochondria and, consequently, there was a compensatory increase in the rate of glycolysis. FCCP depolarized the mitochondrial membrane that increased the OCR because the mitochondria attempted to restore the mitochondrial membrane potential.

\begin{tabular}{llllll}
\hline Antigen & Host & Clonality & Supplier & Code & Dilution \\
\hline CD90 & Rabbit & Monoclonal & Abcam & AB133350 & $1: 100$ \\
CD73 & Mouse & Monoclonal & Abcam & AB54217 & $1: 500$ \\
Oct4 & Rabbit & Polyclonal & Abcam & AB19857 & $1: 400$ \\
CD146 & Rabbit & Monoclonal & Abcam & AB75769 & $1: 100$ \\
CD14 & Mouse & Monoclonal & Abcam & AB181470 & $1: 100$ \\
Goat anti-mouse IgG H\&L alexa fluor 488 & Goat & Polyclonal & Abcam & AB150117 & 1:500 \\
Goat anti-rabbit IgG H\&L alexa fluor 594 & Goat & Polyclonal & Abcam & AB150080 & $1: 500$ \\
\hline
\end{tabular}


Table 2 Seahorse XF cell phenotype test run protocol

\begin{tabular}{llll}
\hline Command & Time $(\min )$ & Port & Drug \\
\hline Calibrate & & & \\
Equilibrate & 12 & & \\
Mix $(\times 5)^{*}$ & 3 & & \\
Measure $(\times 5)^{*}$ & 3 & A & Oligomycin + FCCP \\
Inject & & & \\
Mix $(\times 5)^{* *}$ & 3 & & Hoechst 33342 \\
Measure $(\times 5)^{* *}$ & 3 & B & \\
Inject & & & \\
End protocol & &
\end{tabular}

*After the equilibration, the baseline step consisted of 5 "mix and measure" cycles**After the injection of Oligomycin + FCCP, 5 "mix and measure" cycles were performed

In addition, Hoechst 33342 (Fluka, Biochemika) at the concentration of 1:3000/well was added and the absorbance was measured with EnSpire Multimode Plate Reader $(\mathrm{Ex} / \mathrm{Em}=358 / 461 \mathrm{~nm}$; Perkin Elmer) for the normalization of the Seahorse data. The total run was $1 \mathrm{~h}$ and $12 \mathrm{~min}$ and the run protocol is described in Table 2. Results were exported by using the Seahorse Wave 2.4 XF-96 software and the data obtained were normalized to the absorbance per well and expressed in $\mathrm{pmol} / \mathrm{min} / \mathrm{abs}$.

Table 3 Seahorse XF cell mito stress test run protocol

\begin{tabular}{llll}
\hline Command & Time (min) & Port & Drug \\
\hline Calibrate & & & \\
Equilibrate & 12 & & \\
Mix (x 3)* & 3 & & \\
Measure (x3)* & 3 & Oligomycin \\
Inject & & & \\
Mix (x3)** & 3 & B & FCCP \\
Measure (x3)** & 3 & & \\
Inject & 3 & & Rotenone/Antimycin \\
Mix (x3)** & 3 & C & \\
Measure (x3)** & 3 & & Hoechst 33342 \\
Inject & & D & \\
Mix (x3)** & 3 & & \\
Measure (x3)** & 3 & & \\
Inject & & & \\
End protocol & & & \\
\hline
\end{tabular}

*After the equilibration, the baseline step consisted of 3 "mix and measure" cycles

**After the injection of Oligomycin, FCCP and Rotenone/Antimycin follow 3 "mix and measure" cycles
Table 4 Number of cells at $\mathrm{P} 0$ at confluence

\begin{tabular}{llll}
\hline & Cord 1 & Cord 2 & Cord 3 \\
\hline PV-MSCs & $4.12 \times 10^{6}$ & $2.18 \times 10^{6}$ & $2.075 \times 10^{6}$ \\
WJ-MSCs & $10.7 \times 10^{6}$ & $14.55 \times 10^{6}$ & $4.65 \times 10^{6}$ \\
CL-MSCs & $9.8 \times 10^{6}$ & $7.95 \times 10^{6}$ & $12.5 \times 10^{6}$ \\
\hline
\end{tabular}

\section{Cell Mito Stress Test}

PV-, WJ- and CL-MSCs were seeded into the Seahorse XF96 Cell Culture Microplates (Agilent) at the cell density of 10,000 cells $/ \mathrm{cm}^{2}$ and cultured until confluence. The cells were exposed to $\mathrm{OGD} / \mathrm{R}$ as described above. Prior to the start of the Seahorse XF Cell Energy Phenotype test, a sensor cartridge was hydrated in Seahorse XF Calibrant (Agilent) following the manufacturer's instruction (Agilent). The day of the assay, the cells were washed one time and incubated in XF Seahorse Base Medium DMEM (Agilent) supplemented with $5.5 \mathrm{mM}$ glucose, $1 \mathrm{mM}$ sodium pyruvate, and $4 \mathrm{mM}$ L-glutamine and 1x NEAA to mimic both cell growth and reperfusion conditions after OGD. The XF Seahorse Base Medium DMEM was prepared following the manufacturer's instruction (Agilent). For both control and $\mathrm{OGD} / \mathrm{R}$ conditions, OCR and ECAR readings were taken over time under basal conditions and after the addition of mitochondrial inhibitors $(1 \mu \mathrm{M}$ oligomycin, $1 \mu \mathrm{M}$ FCCP and $0.5 \mu \mathrm{M}$ rotenone/ antimycin).

In addition, Hoechst 33342 (Fluka, Biochemika) was added at the concentration of 1:3000/well. The absorbance was measured with EnSpire Multimode Plate Reader $(E x / E m=358 / 461 \mathrm{~nm}$; Perkin Elmer $)$ and the nuclei count was performed with a fluorescent phase contrast microscope (Olympus) for the normalization of the Seahorse data.

The total run was $1 \mathrm{~h}$ and $24 \mathrm{~min}$ and the run protocol is described in Table 3. Results were exported by using the Seahorse Wave 2.4 XF-96 software and the data obtained for each condition were normalized to the cell number per well and expressed in $\mathrm{pmol} / \mathrm{min} /$ cells.

Table 5 Immunocytochemistry results of markers expression

\begin{tabular}{llllll}
\hline & CD90 & CD73 & CD146 & Oct4 & CD14 \\
\hline PV-MSC & ++ & +++ & +++ & +++ & ++ \\
WJ-MSC & +++ & ++ & +++ & +++ & ++ \\
CL-MSC & +++ & ++ & +++ & ++ & ++ \\
\hline
\end{tabular}

Results of the immunocytochemical analysis are represented semiquantitatively. Frequency of cells (\#): $+=\#<33 \% ;++=33 \%<\#<66 \% ;+++$ $=\#>66 \%$ ) 
Fig. 1 Morphological features of the three MSC populations of the $\mathrm{UC}$ at P0. Magnification 20x, bar $50 \mu \mathrm{m}$

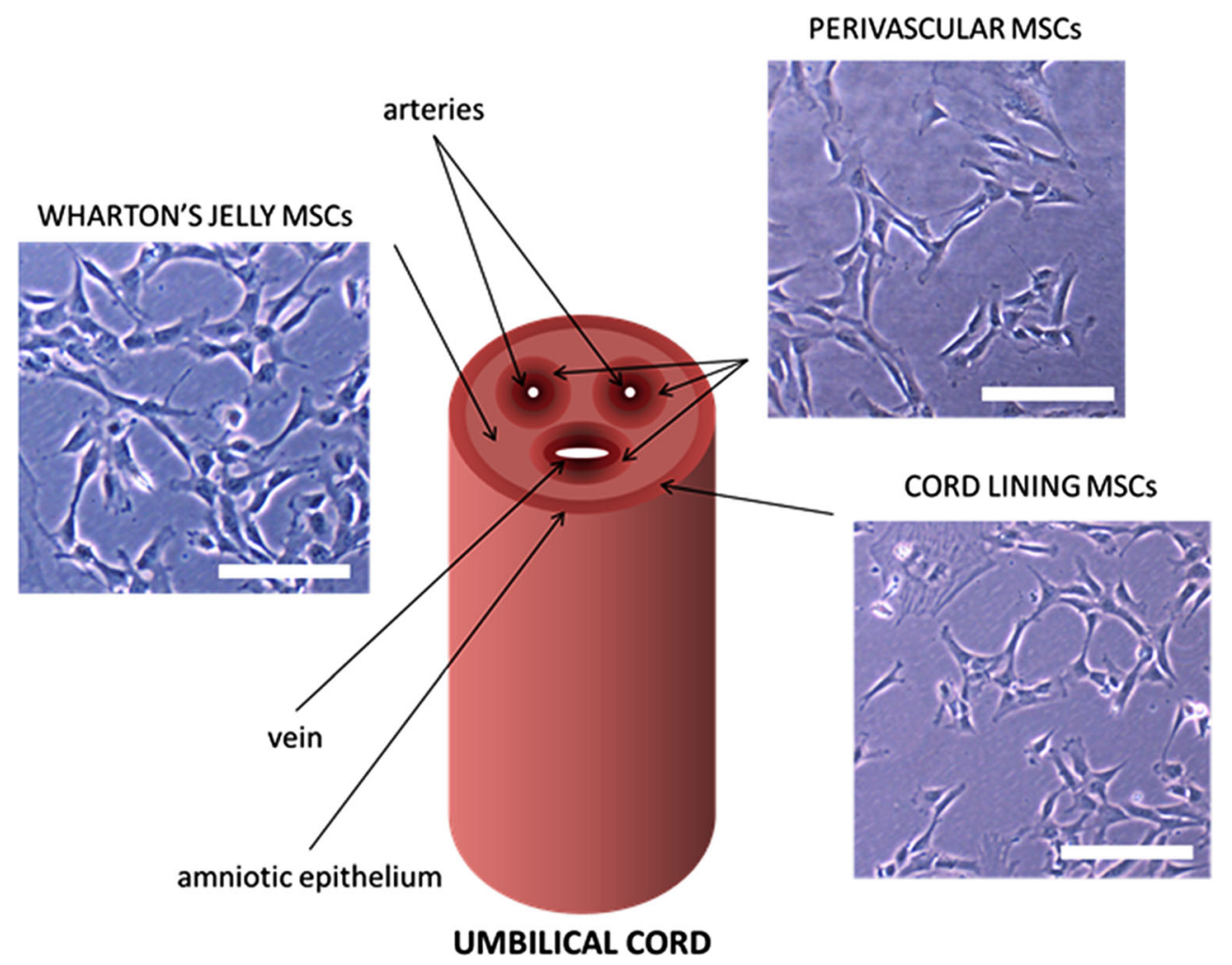

\section{Statistical Analysis}

All statistic analyses were performed using GraphPad Prism software (https://www.graphpad.com/scientificsoftware/prism/). Data are shown as mean \pm SEM. Statistical analysis was performed with either Mann Whitney or Wilcoxon tests.

\section{Results}

\section{Characterization of the Three MSCs Populations of UC}

In view of accumulating evidence of the existence of three different populations of MSCs in hUC as well as the necessity of a phenotypic characterization with a greater clarity of their properties, we isolated PV-, WJand CL-MSCs from UC as described in the Methods section. The number of cells obtained at P0 from the tested hUCs is shown in Table 4. The morphological analysis is shown in Fig. 1. Immunofluorescence staining showed that they all expressed mesenchymal markers CD90 and CD73 (Fig. 2). In addition, PVMSCs expressed CD146, WJ-MSCs expressed Oct4 and CL-MSCs expressed CD14 (Fig. 2), according with previous findings by our group and others $2012[15,16$, $18,19]$. In addition, the results of the immunocytochemical analysis are represented semiquantitatively in Table 5.

\section{Mitochondrial Function of PV-, WJ- and CL-MSCs}

In order to understand the metabolic energy profile and mitochondrial function of PV-, WJ- and CL-MSCs, Cell Energy Phenotype and Cell Mito Stress tests were performed by using the Seahorse XF96 Analyzer. Our overall goal was to analyze the metabolic energy profile of the three hUC-MSCs populations.

The XF Cell Energy Phenotype Test (Fig. 3) revealed that all three hUC-MSCs populations displayed a comparable metabolic phenotype with significant increases of metabolic response with respect to baseline (Fig. 3C, $p=0.0005$; Fig. 3D, PV-MSC $\mathrm{p}<0.0001$, WJ-MSC $\mathrm{p}<0.0001$, CL-MSC $\mathrm{p}<$ 0.0001 ; Fig. 3E, PV-MSC $p<0.0001$, WJ-MSC $p=0.0001$, CL-MSC $p<0.0001$ ). In addition, our data suggest that the metabolic profile of PV-, WJ- and CL-MSCs can be classified as "quiescent". This is evidenced by undifferentiated MSCs exhibiting low levels of mitochondrial activities and low levels of glycolytic activities [20].

In order to assay the mitochondrial function of PV-, WJand CL-MSCs in ischemic/reperfused conditions, a Cell Mito Stress test was performed on both controls and cells subjected 
Fig. 2 Immunolocalization of CD90, CD73, Oct4, CD146 and CD14. Magnification 20x, bar 50 $\mu \mathrm{m}$. Immunofluorescence staining reveals that the cells all expressed MSC markers (CD90 and CD73). In addition, PV-, WJand CL-MSCs expressed CD146, Oct4 and CD14, respectively. The nuclei were stained with DAPI

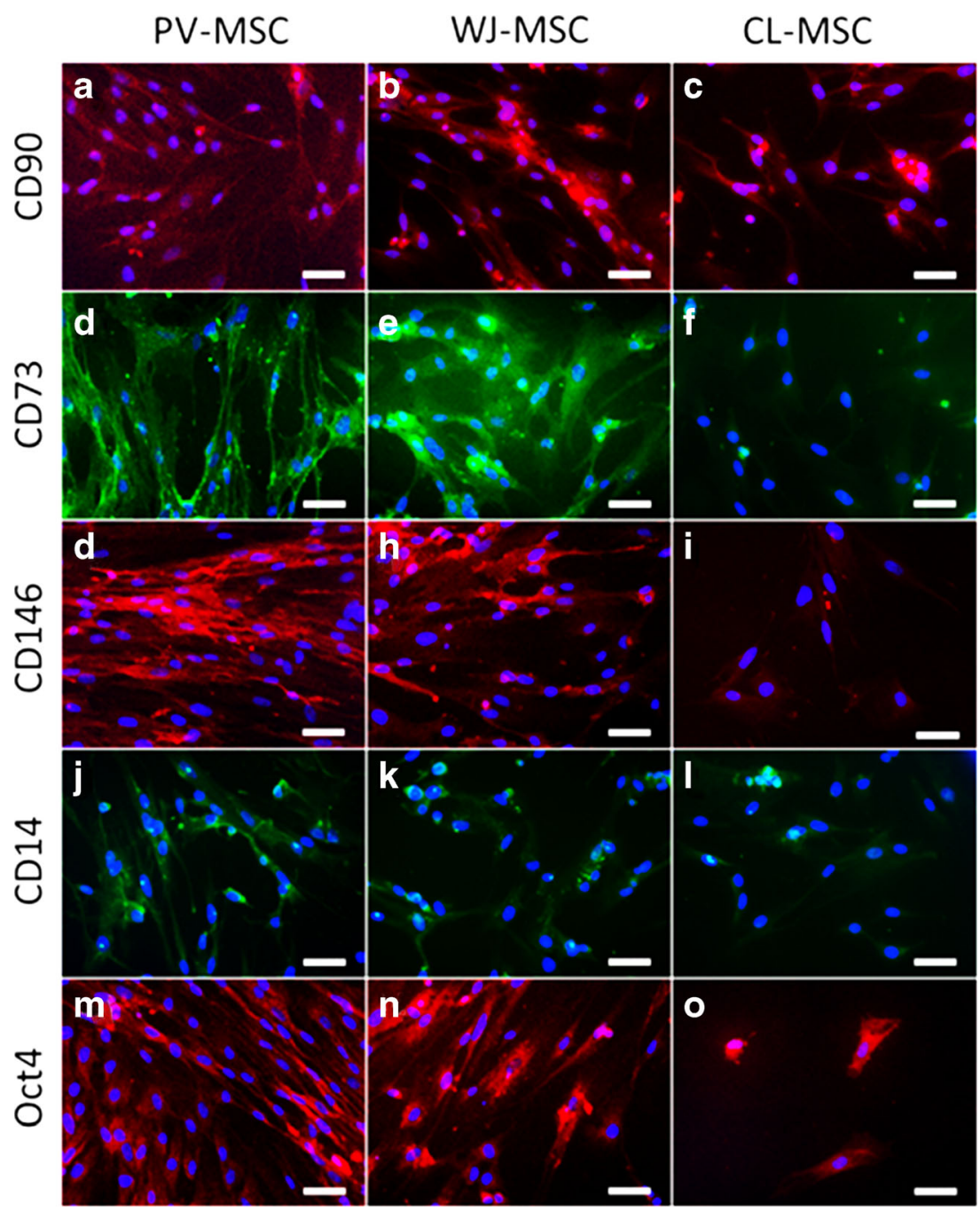

to OGD/R (Fig. 4). Seahorse XF Cell Mito Stress test showed that all three hUC-MSCs populations exhibited comparable mitochondrial respiration parameters in both control and OGD/R conditions, demonstrating their ability to survive in ischemic/reperfused conditions (Fig. 4B, PV-MSC p = 0.0041; Fig. 4C, PV-MSC $\mathrm{p}=0.0023$; Fig. 4E PV-MSC $\mathrm{p}=$ 0.0012; Fig. 4F, PV-MSC $\mathrm{p}=0.0070$, WJ-MSC $\mathrm{p}=0.0293$ ).

In addition, a cell viability test showed that all three hUCMSCs cellular populations showed good survival and maintained their proliferation following OGD/R, further suggesting their ability to survive in ischemic/reperfused conditions (Fig. 5 , PV-MSC $\mathrm{p}=0.0005$; WJ-MSC $\mathrm{p}=0.0198)$. These results demonstrate the adaptive capacity of PV-, WJ- and CL-MSCs under $\mathrm{OGD} / \mathrm{R}$ conditions. This capacity to survive is probably due to their robust mitochondrial function. In particular, the OGD/R condition did not affect WJ-MSC and CL-MSC metabolism and viability (Figs. 4 and 5). Although OGD/R condition significantly altered PV-MSC metabolism, their cell viability remained robust (Figs. 4 and 5). In summary, the three populations of hUC-MSCs could be a potential source of mitochondria-based stem cell therapy for stroke.

\section{Discussion}

Mitochondria dysfunction is a direct consequence of oxygen and glucose deprivation during stroke that contributes to oxidative stress, neuronal death and inflammation [2]. Emerging evidence of mitochondria transfer from stem cells to ischemic cells paved the way for mitochondrial-based stem cell therapy of stroke $[6,7,10]$. hUCs received only three blood vessels suggesting that hUC-MSCs are normally adapted to survive in a relatively hypoxic and glucose-poor environment, thus these cells represent an attractive source for stem cell-based therapy in ischemic pathologies, such as stroke [13]. Although several studies have shown that the regulation of energy metabolism is critical for MSC functions and their proliferation and differentiation dynamics, few studies have been focused on the 


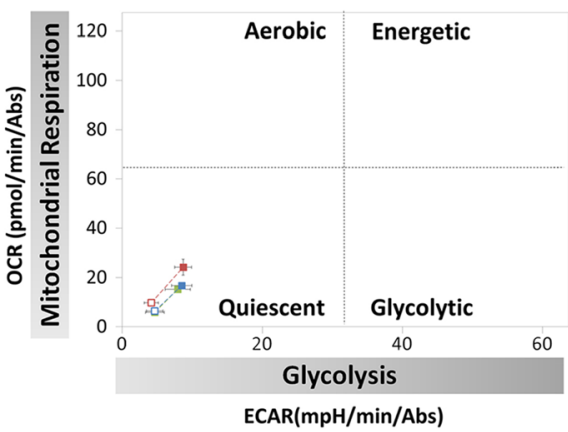

$-P V-M S C$

- WJ-MSC b

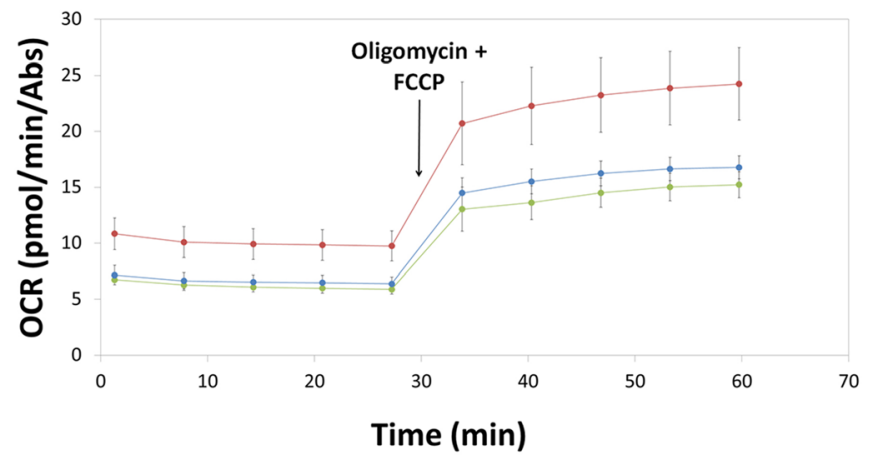

-PV-MSC $\quad$ WJ-MSC $\quad \cdot$ CL-MSC
C

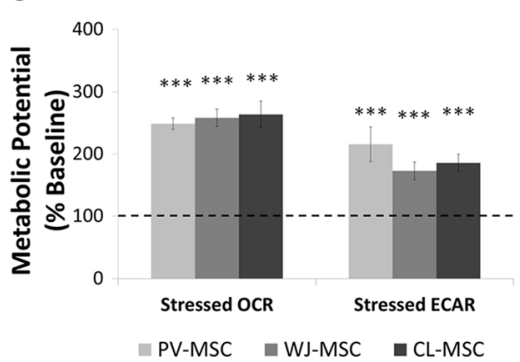

d

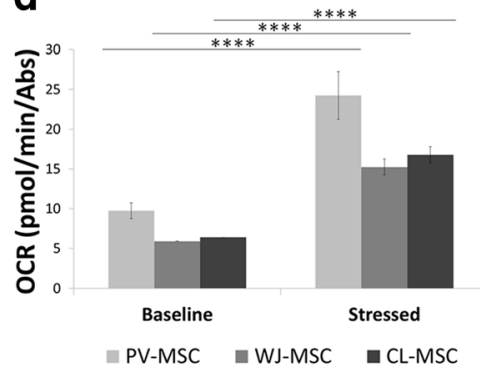

e

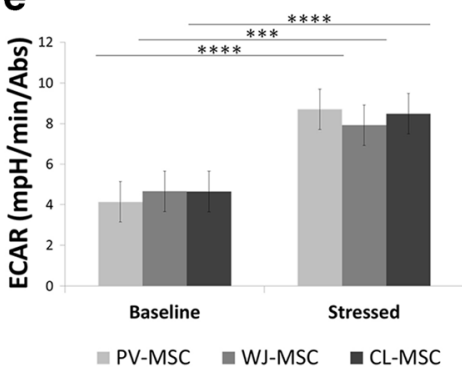

Fig. 3 Seahorse XF Cell Energy Phenotype Test performed by using a Seahorse XF96 Analyzer ( $\mathrm{n}=12$ for each cell type). Oligomycin $1 \mu$ M, FCCP 1 $\mu \mathrm{M}$. OCR: oxygen consumption rate. ECAR: extracellular acidification rate. $* * * \mathrm{P}<0.001$; **** $\mathrm{P}<0.0001$ vs. Baseline

energy metabolism and mitochondrial function of hUC-MSCs [20-22]. In addition, growing evidence suggests the existence of three different populations of hUC-MSCs (PV-, WJ- and CL-MSCs) [13]. However, whether a population is superior to another is not still clear [23]. Likewise, whether the different anatomical distance from the vessels of the three MSCs population in the UC might result in a different energy metabolism and in a growing resistance and survival capacity in a poor oxygen and glucose environment has not been investigated.

With this gap in knowledge, PV-, WJ- and CL-MSCs were isolated from hUCs in order to understand (1) the energy metabolism profile, (2) the mitochondrial function and (3) their survival capacity in both normal and after ischemic/reperfused conditions. Studies in bone marrow-derived -MSCs showed that these cells display a glycolytic metabolism in undifferentiated state, while a switch to OXPHOS metabolism occurred during the differentiation [20]. These previous studies were performed using different methods including measurement of mitochondrial mass, determination of mtDNA copy number, western blot analysis of glycolytic and mitochondrial enzymes, assay of the expression of mitochondrial biogenesisassociated genes, assessment of intracellular ATP content, OCR measurement using the 782 OxygenMeter and quantization of radioactive labeled glucose [20, 24, 25]. Few studies have used the Seahorse analyzer for OCR measurement in differentiated AD-MSCs and iPSC-derived mesenchymal progenitor cells [26-28]. A study analyzed the hUC-MSCs metabolism by measuring the dissolved $\mathrm{O}_{2}$ and $\mathrm{pH}$ values in the culture medium using the SFR-Shake Flask Reader [21].

Here, for the first time, the energy metabolism of the three populations of hUC-MSCs was analyzed by using the Seahorse Analyzer, which allows a sensitive kinetic measure of OCR, and ECAR in live cells and in real time. OCR is the rate of the decrease of oxygen concentration in the assay medium and, therefore, it is an indicator of mitochondrial respiration. ECAR corresponds to the rate of increase in proton concentration (or decrease in $\mathrm{pH}$ ) in the assay medium and, therefore, it is a measure of the rate of glycolysis. In this study, the cell energy phenotype test and cell mito stress test assays were performed by using the Seahorse Analyzer (Figs. 3 and 4). The results of the cell energy phenotype test showed that PV-, WJ- and CLMSCs are characterized by a similar energy metabolism (Fig. 3). In particular, the three MSC types exhibited a quiescent phenotype. Thus, PV-, WJ- and CL-MSCs maintained both mitochondrial and glycolytic activities at low levels.

Under stress conditions (Oligomycin + FCCP injection) the three types of MSCs displayed an increase in the glycolytic pathway likely to balance the reduction of mitochondrial respiration. Moreover, the Seahorse cell mito stress test was 

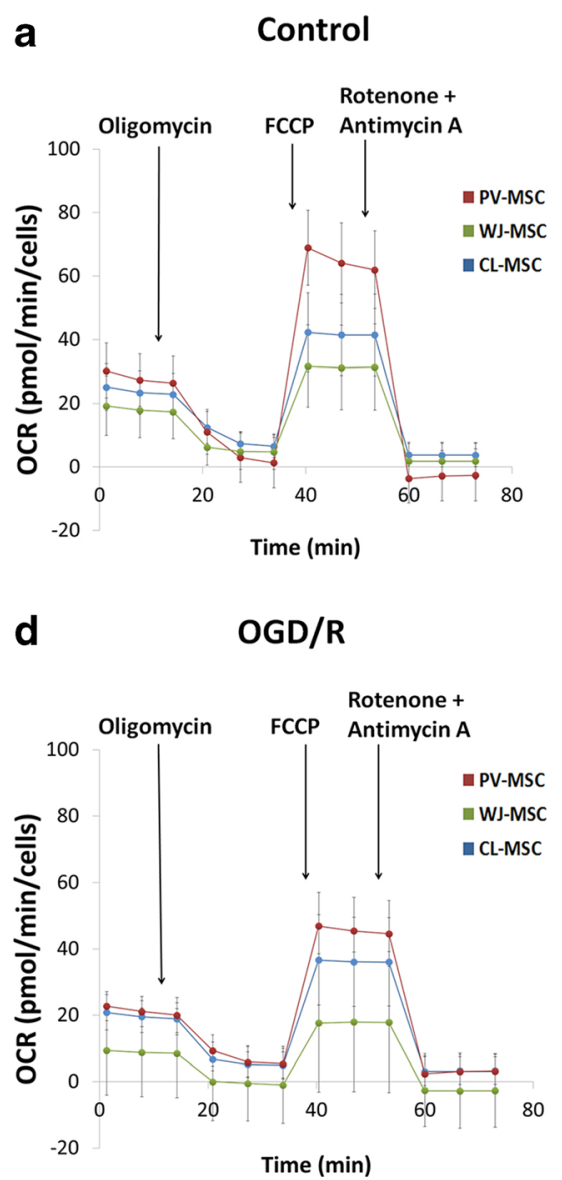

Fig. 4 Seahorse XF Mito Stress test shows PV-, WJ- and CL-MSCs in both normal and after OGD/R conditions ( $\mathrm{n}=07$ for PV-MSC and CLMSC; $\mathrm{n}=08$ for WJ-MSC control; $\mathrm{n}=06$ for WJ-MSC OGD/R). OCR:

performed in both normal and after OGD/R to analyze the mitochondrial activity of PV-, WJ- and CL-MSCs in ischemic/reperfused conditions (Fig. 4). Although the OCR exhibited a slight decrease in $\mathrm{OGD} / \mathrm{R}$ groups, all the three hUC-MSC populations showed a comparable robust resistance and adaption to ischemic/reperfused conditions as also demonstrated by the cell viability test. Indeed, the survival of cells was not affected by OGD/R treatment but surprisingly the number of the cells increased suggesting a powerful ability of all three types of hUC-MSCs to survive in stroke conditions (Fig. 5).

That MSCs augment mitochondria function may play a key role in sequestering the mitochondrial dysfunction implicated in stroke-induced secondary cell death [29-31]. The isolation of potent hUC-MSCs [32-39] towards repairing the mitochondria stands as novel stroke therapy. Cell-based regenerative medicine, which has been demonstrated to be a potent treatment for a number of neurological disorders [39-46], may benefit from transplantation of stem cell-derived mitochondria.

Taken together, these results demonstrate the adaptive capacity of PV-, WJ- and CL-MSCs to ischemic
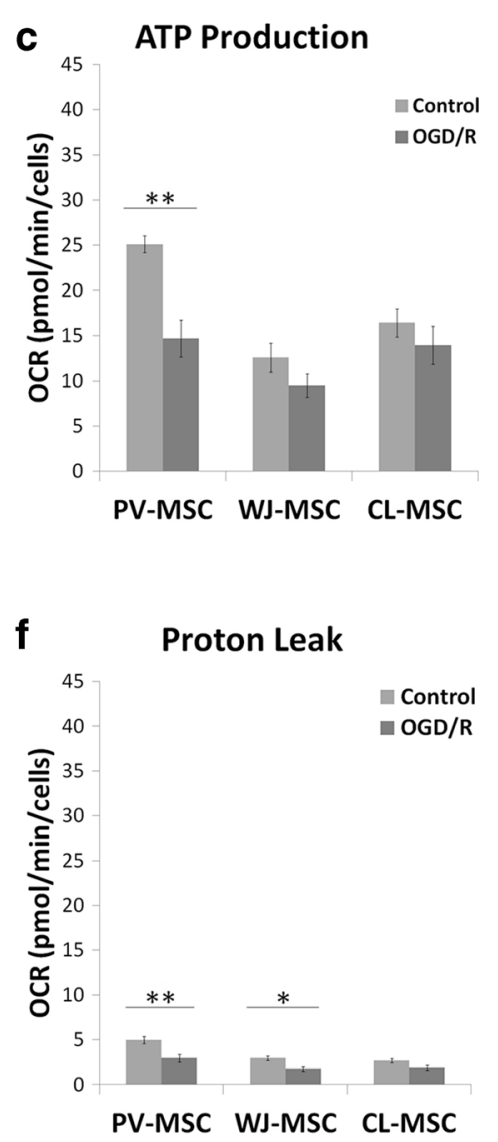

oxygen consumption rate. $1 \mu \mathrm{M}$ Oligomycin, $1 \mu \mathrm{M}$ FCCP, $0.5 \mu \mathrm{M}$ Rotenone + Antimycin A. **P $<0.01 ; * \mathrm{P}<0.05$

environments due to their maintained mitochondrial function. All three types of hUC-MSCs displayed a similar energy metabolism and mitochondrial function. PVMSCs showed the highest OCR values in both Seahorse tests suggesting a superior mitochondrial activity in these cells compared to the other hUC-MSC populations. A further support of this result is the more consistent reduction of OCR of PV-MSCs after OGD/R compared to WJand CL-MSCs (Fig. 4). CL-MSCs were the cells least affected by OGD/R condition (Fig. 4), suggesting their robust survival in ischemic environment. Further investigations are needed to better understand whether these slight but significant differences among the three hUCMSCs are due to the specific region's composition of different number of healthy mitochondria or improved adaptation of mitochondria to ischemic conditions. Despite these limitations, hUC-MSCs appear to tolerate the nonconducive cellular environment and continue to display viable and functional mitochondriaafter ischemic/ reperfusion injury. Thus, the three populations of hUCMSCs stand as promising cell source for mitochondriabased stem cell therapy of stroke. 

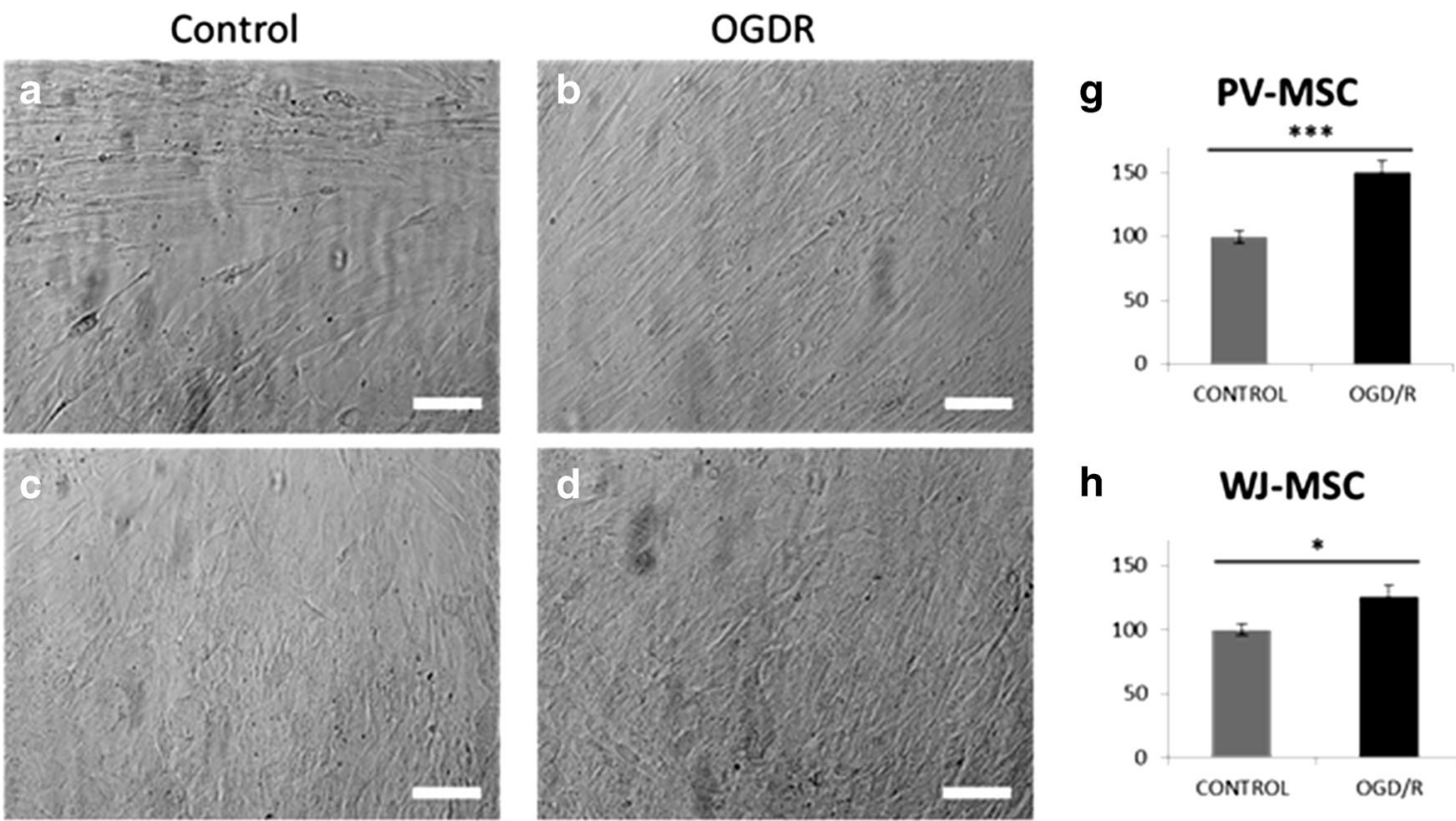

h WJ-MSC
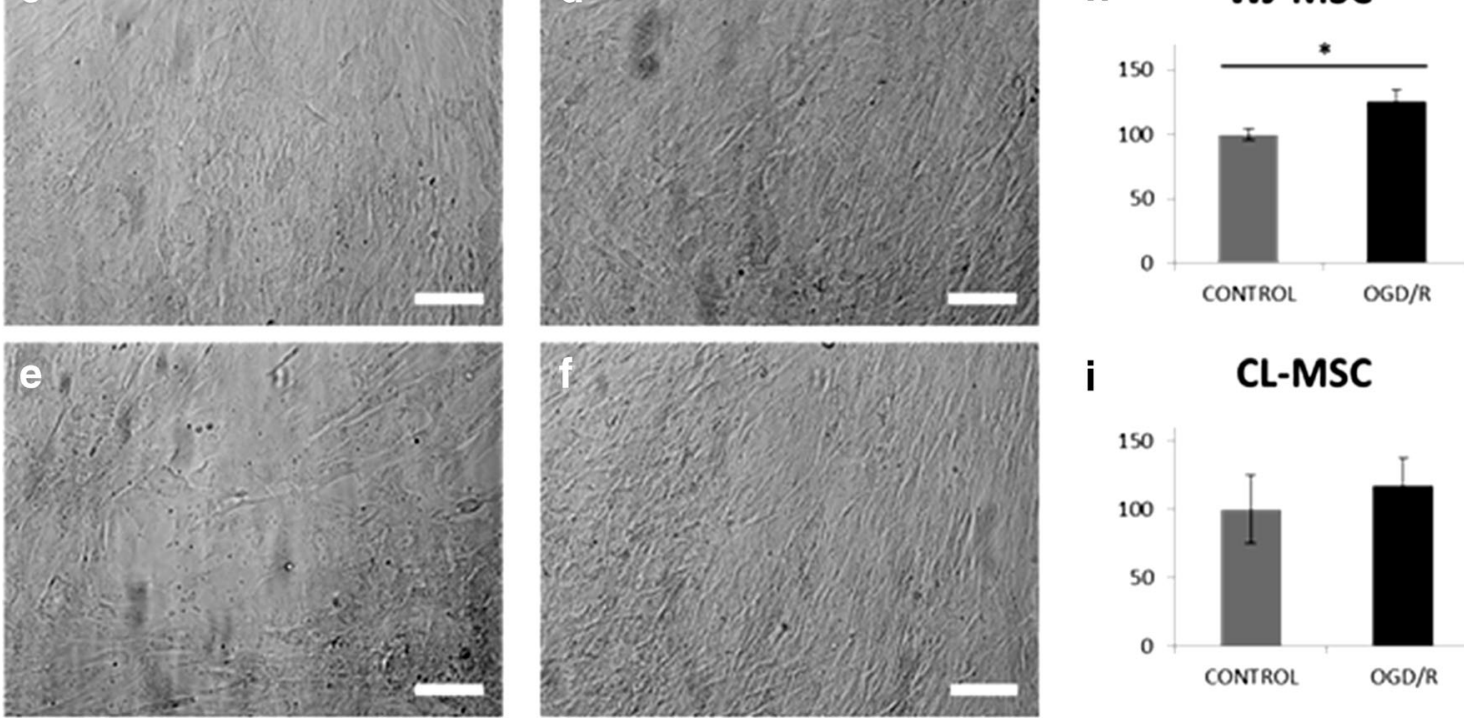

i

\section{CL-MSC}

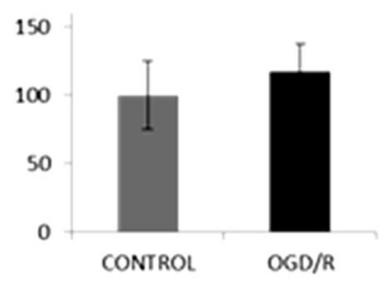

Fig. 5 Cell viability tested by using Calcein AM stain in both control and after OGD/R conditions ( $\mathrm{n}=21$ for each cell type). Magnification $10 \mathrm{x}$, bar 100 $\mu \mathrm{M}$. $* * * \mathrm{P}<0.001 ; * \mathrm{P}<0.05$

\section{Conclusions}

Energy metabolism assay via the Seahorse technology revealed for the first time that PV-MSCs, WJ- MSCs, and CL-MSCs of the hUC displayed a robust mitochondrial profile with great capacity of survival under ischemic conditions suggesting that these hUC-MSCs represent an effective source of donor cells for mitochondria-based stem cell therapy in stroke and likely other ischemic disorders.

Acknowledgements We thank the entire staff of Borlongan Neural Transplantation Laboratory for technical assistance and excellent scientific discussion.

Funding Information Dr Borlongan is funded by National Institutes of Health (NIH) R01NS090962, NIH R01NS102395, and NIH R21NS109575.

\section{Compliance with Ethical Standards}

Disclosure of Potential Conflicts of Interest Dr Borlongan is funded and received royalties and stock options from Astellas, Asterias, Sanbio,
Athersys, KMPHC, and International Stem Cell Corporation; and also received consultant compensation for Chiesi Farmaceutici. He also holds patents and patent applications related to stem cell biology and therapeutic applications.

Research Involving Human Participants and/or Animals No human participants or animals were involved in this study.

Informed Consent Not applicable.

Open Access This article is licensed under a Creative Commons Attribution 4.0 International License, which permits use, sharing, adaptation, distribution and reproduction in any medium or format, as long as you give appropriate credit to the original author(s) and the source, provide a link to the Creative Commons licence, and indicate if changes were made. The images or other third party material in this article are included in the article's Creative Commons licence, unless indicated otherwise in a credit line to the material. If material is not included in the article's Creative Commons licence and your intended use is not permitted by statutory regulation or exceeds the permitted use, you will need to obtain permission directly from the copyright holder. To view a copy of this licence, visit http://creativecommons.org/licenses/by/4.0/. 


\section{References}

1. Benjamin, E. J., Virani, S. S., Callaway, C. W., Chamberlain, A., Chang, A. R., Cheng, S. et al. (2018). Heart disease and stroke statistics-2018 update: a report from the American Heart Association. Circulation, 137(12), e67-e492. https://doi.org/10. 1161/CIR.0000000000000558

2. Yang, J. L., Mukdaab, S., \& Chen, S. D. (2018). Diverse roles of mitochondria in ischemic stroke. Redox Biology, 16, 263-275. https://doi.org/10.1016/j.redox.2018.03.002

3. Watts, L. T., Lloyd, R., Garling, R. J., \& Duong, T. (2013). Stroke neuroprotection: targeting mitochondria. Brain Sciences, 3(2), 540560. https://doi.org/10.3390/brainsci3020540

4. Tajiri, N., Duncan, K., Antoine, A., Pabon, M., Acosta, S. A., de la Pena, I., \& Borlongan, C. V. (2014). Stem cell-paved biobridge facilitates neural repair in traumatic brain injury. Frontiers in Systems Neuroscience, 8(116), 1-6. https://doi.org/10.3389/fnsys. 2014.00116

5. Caprnda, M., Kubatka, P., Gazdikova, K., Gasparova, I., Valentova, V., Stollarova, N. et al. (2017). Immunomodulatory effects of stem cells: therapeutic option for neurodegenerative disorders. Biochemical Pharmacology, 91(2017), 60-69. https://doi.org/10. 1016/j.biopha.2017.04.034

6. Russo, E., Napoli, E., \& Borlongan, C. V. (2018). Healthy mitochondria for stroke cells. Brain Circulation, 4(3), 95-98. https://doi. org/10.4103/bc.bc_20_18

7. Russo, E., Nguyen, H., Lippert, T., Tuazon, J., Borlongan, C. V., \& Napoli, E. (2018). Mitochondrial targeting as a novel therapy for stroke. Brain Circulation, 4(3), 84-94. https://doi.org/10.4103/bc. bc 1418

8. Hsu, Y. C., Wu, Y. T., Yu, T. H., \& Wei, Y. H. (2016). Mitochondria in mesenchymal stem cell biology and cell therapy: from cellular differentiation to mitochondrial transfer. Seminars in Cell and Developmental Biology, 52, 119-131. https://doi.org/10.1016/j. semcdb.2016.02.011

9. Paliwal, S., Chaudhuri, R., Agrawal, A., \& Mohanty, S. (2018). Regenerative abilities of mesenchymal stem cells through mitochondrial transfer. Journal of Biomedical Science, 25(1), 31. https://doi.org/10.1186/s12929-018-0429-1

10. Borlongan, C. V., Nguyen, H., Lippert, T., Russo, E., Tuazon, J., $\mathrm{Xu}, \mathrm{K}$. et al. (2018). May the force be with you: transfer of healthy mitochondria from stem cells to stroke cells. Journal of Cerebral Blood Flow and Metabolism, 39(2), 367-370. https://doi.org/10. 1177/0271678X18811277

11. La Rocca, G., Lo Iacono, M., Corsello, T., Corrao, S., Farina, F., \& Anzalone, R. (2013). Human Wharton's jelly mesenchymal stem cells maintain the expression of key immunomodulatory molecules when subjected to osteogenic, adipogenic and chondrogenic differentiation in vitro: new perspectives for cellular therapy. Current Stem Cell Research \& Therapy, 8(1), 100-113. https://doi.org/10. 2174/1574888X11308010012

12. Magatti, M., Abumaree, M. H., Silini, A. R., Anzalone, R., Saieva, S., Russo, E., et al. (2016). Chapter 6. The Immunomodulatory Features of Mesenchymal Stromal Cells Derived from Wharton's Jelly, Amniotic Membrane, and Chorionic Villi: In Vitro and In Vivo Data. In O. Parolini (A cura di), Placenta: The Tree of Life (p. 91-128). https://doi.org/10.1201/b19620-11.

13. Davies, J. E., Walker, J. T., \& Keating, A. (2017). Concise review: Wharton's jelly: the rich, but enigmatic, source of mesenchymal stromal cells. Stem Cells Translational Medicine, 6(7), 16201630. https://doi.org/10.1002/sctm.16-0492

14. Mennan, C., Wright, K., Bhattacharjee, A., Balain, B., Richardson, J., \& Roberts, S. (2013). Isolation and characterisation of mesenchymal stem cells from different regions of the human umbilical cord. Biotechnology Research International, 2013, 916136. https:// doi.org/10.1155/2013/916136

15. Sarugaser, R., Ennis, J., Stanford, W. L., \& Davies, J. E. (2009). Isolation, propagation, and characterization of human umbilical cord perivascular cells (HUCPVCs). Methods in Molecular Biology, 482, 269-279. https://doi.org/10.1007/978-1-59745-060717

16. Kita, K., Gauglitz, G. G., Phan, T. T., Herndon, D. N., \& Jeschke, M. G. (2010). Isolation and characterization of mesenchymal stem cells from the sub-amniotic human umbilical cord lining membrane. Stem Cells and Development, 19(4), 491-502. https://doi. org $/ 10.1089 / \mathrm{scd} .2009 .0192$

17. Kaneko, Y., Tajiri, N., Shojo, H., \& Borlongan, C. V. (2014). Oxygen-glucose-deprived rat primary neural cells exhibit DJ-1 translocation into healthy mitochondria: a potent stroke therapeutic target. CNS Neuroscience and Therapeutics, 20(3), 275-281. https://doi.org/10.1111/cns.12208

18. La Rocca, G., Anzalone, R., Corrao, S., Magno, F., Loria, T., Iacono, L. et al. (2009). Isolation and characterization of Oct-4+/ HLA-G + mesenchymal stem cells from human umbilical cord matrix: differentiation potential and detection of new markers. Histochemistry and Cell Biology, 131(2), 267-282. https://doi. org/10.1007/s00418-008-0519-3

19. Shaikh, A., \& Bhartiya, D. (2012). Pluripotent stem cells in bone marrow and cord blood. In T. E. Moschandreou (Ed.), Blood Cell An Overview of Studies in Hematology (pp. 69-88). https://doi.org/ $10.5772 / 48133$.

20. Hu, C., Fan, L., Cen, P., Chen, E., Jiang, Z., \& Li, L. (2016). Energy metabolism plays a critical role in stem cell maintenance and differentiation. International Journal of Molecular Sciences, 17(2), 253. https://doi.org/10.3390/ijms17020253

21. Lavrentieva, A., Majore, I., Kasper, C., \& Hass, R. (2010). Effects of hypoxic culture conditions on umbilical cord-derived human mesenchymal stem cells. Cell Communication and Signaling: CCS, 8 (18). https://doi.org/10.1186/1478-811X-8-18.

22. Skiles, M. L., Brown, K. S., Tatz, W., Swingle, K., \& Brown, H. L. (2018). Quantitative analysis of composite umbilical cord tissue health using a standardized explant approach and an assay of metabolic activity. Cytotherapy, 20(4), 564-575. https://doi.org/10. 1016/j.jcyt.2018.01.001

23. Conconi, M. T., Di Liddo, R., Tommasini, M., Calore, C., \& Parnigotto, P. P. (2011). Phenotype and differentiation potential of stromal populations obtained from various zones of human umbilical cord: an overview. The Open Tissue Engineering and Regenerative Medicine Journal, 4, 6-20. https://doi.org/10.2174/ 1875043501104010006

24. Chen, C. T., Shih, Y. R., Kuo, T. K., Lee, O. K., \& Wei, Y. H. (2008). Coordinated changes of mitochondrial biogenesis and antioxidant enzymes during osteogenic differentiation of human mesenchymal stem cells. Stem Cells, 26(4), 960-968. https://doi.org/10. 1634/stemcells.2007-0509

25. Fillmore, N., Huqi, A., Jaswal, J. S., Mori, J., Paulin, R., Haromy, A. et al. (2015). Effect of fatty acids on human bone marrow mesenchymal stem cell energy metabolism and survival. PLoS One, 10(3), e0120257. https://doi.org/10.1371/journal.pone.0120257

26. Balhara, B., Burkart, A., Topcu, V., Lee, Y. K., \& Cowan, C. K. (2015). Severe insulin resistance alters metabolism in mesenchymal progenitor cells. Endocrinology, 156(6), 2039-2048. https://doi. org/10.1210/en.2014-1403

27. Moisan, A., Lee, Y. K., Zhang, J. D., Hudak, C. S., Meyer, C. A., Prummer, M. et al. (2015). White-to-brown metabolic conversion of human adipocytes by JAK inhibition. Nature Cell Biology, 17(1), 57-67. https://doi.org/10.1038/ncb3075

28. van den Beukel, J. C., Grefhorst, A., Hoogduijn, M. J., Steenbergen, J., Mastroberardino, P. G., Dor, F. J. et al. (2015). Women have more potential to induce browning of perirenal 
adipose tissue than men. Obesity (Silver Spring, Md.), 23(8), 16711679. https://doi.org/10.1002/oby.21166

29. Stimpfel, M., Jancar, N., \& Virant-Klun, I. (2018). New challenge: mitochondrial epigenetics? Stem Cell Reviews and Reports, 14(1), 13-26. https://doi.org/10.1007/s12015-017-9771-z

30. Kornicka, K., Houston, J., \& Marycz, K. (2018). Dysfunction of mesenchymal stem cells isolated from metabolic syndrome and type 2 diabetic patients as result of oxidative stress and autophagy may limit their potential therapeutic use. Stem Cell Reviews and Reports, 14(3), 337-345. https://doi.org/10.1007/s12015-018-9809-x

31. Aghajani Nargesi, A., Zhu, X. Y., Hickson, L. J., Conley, S. M., van Wijnen, A. J., Lerman, L. O., \& Eirin, A. (2019). Metabolic syndrome modulates protein import into the mitochondria of porcine mesenchymal stem cells. Stem Cell Reviews and Reports, 15(3), 427-438. https://doi.org/10.1007/s12015-018-9855-4

32. Al Naem, M., Bourebaba, L., Kucharczyk, K., Röcken, M., \& Marycz, K. (2019). Therapeutic mesenchymal stromal stem cells: Isolation, characterization and role in equine regenerative medicine and metabolic disorders. Stem Cell Reviews and Reports. https:// doi.org/10.1007/s12015-019-09932-0.

33. Nishida, F., Zappa Villar, M. F., Zanuzzi, C. N., Sisti, M. S., Camiña, A. E., Reggiani, P. C., \& Portiansky, E. L. (2019). Intracerebroventricular delivery of human umbilical cord mesenchymal stem cells as a promising therapy for repairing the spinal cord injury induced by Kainic acid. Stem Cell Reviews and Reports. https://doi.org/10.1007/s12015-019-09934-y.

34. Contentin, R., Demoor, M., Concari, M., Desancé, M., Audigié, F., Branly, T., \& Galéra, P. (2019). Comparison of the chondrogenic potential of mesenchymal stem cells derived from bone marrow and umbilical cord blood intended for cartilage tissue engineering. Stem Cell Reviews and Reports. https://doi.org/10.1007/s12015-019-09914-2.

35. Corsello, T., Amico, G., Corrao, S., Anzalone, R., Timoneri, F., Lo Iacono, M., et al. (2019). Wharton's jelly mesenchymal stromal cells from human umbilical cord: a close-up on immunomodulatory molecules featured in situ and in vitro. Stem Cell Reviews and Reports, 15(6), 900-918. https://doi.org/10.1007/s12015-019-09907-1

36. Lee, J. Y., Tuazon, J. P., Corey, S., Bonsack, B., Acosta, S., Ehrhart, J., et al. (2019). A gutsy move for cell-based regenerative medicine in Parkinson's disease: targeting the gut microbiome to sequester inflammation and neurotoxicity. Stem Cell Reviews and Reports, 15(5), 690-702. https://doi.org/10.1007/s12015-019-09906-2

37. Lehmann, M., Zappa-Villar, M. F., García, M. G., Mazzolini, G., Canatelli-Mallat, M., Morel, G. R., et al. (2019). Umbilical cord cell therapy improves spatial memory in aging rats. Stem Cell Reviews and Reports, 15(4), 612-617. https://doi.org/10.1007/s12015-019-09895-2

38. Kong, C. M., Subramanian, A., Biswas, A., Stunkel, W., Chong, Y. S., Bongso, A., \& Fong, C. Y. (2019). Changes in stemness properties, differentiation potential, oxidative stress, senescence and mi- tochondrial function in Wharton's jelly stem cells of umbilical cords of mothers with gestational diabetes mellitus. Stem Cell Reviews and Reports, 15(3), 415-426. https://doi.org/10.1007/s12015-0199872-y

39. Borlongan, C. V., Su, T. P., \& Wang, Y. (2000). Treatment with delta opioid peptide enhances in vitro and in vivo survival of rat dopaminergic neurons. Neuroreport, 11(5), 923-6. https://doi.org/ 10.1097/00001756-200004070-00005

40. Borlongan, C. V., Chopp, M., Steinberg, G. K., Bliss, T. M., Li, Y., $\mathrm{Lu}$, M., et al. (2008). Potential of stem/progenitor cells in treating stroke: the missing steps in translating cell therapy from laboratory to clinic. Regenerative Medicine, 3(3), 249-50. https://doi.org/10. 2217/17460751.3.3.249

41. Borlongan, C. V., Stahl, C. E., Cameron, D. F., Saporta, S., Freeman, T. B., Cahill, D. W., \& Sanberg, P. R. (1996). CNS immunological modulation of neural graft rejection and survival. Neurological Research, 18(4), 297-304. https://doi.org/10.1080/ 01616412.1996 .11740425

42. Xia, C. F., Yin, H., Borlongan, C. V., Chao, J., \& Chao, L. (2004). Adrenomedullin gene delivery protects against cerebral ischemic injury by promoting astrocyte migration and survival. Human Gene Therapy, 15(12), 1243-1254. https://doi.org/10.1089/hum. 2004.15.1243

43. Borlongan, C. V., Sanberg, P. R., \& Freeman, T. B. (1999). Neural transplantation for neurodegenerative disorders. Lancet, 353(Suppl 1), SI29-S30. https://doi.org/10.1016/s0140-6736(99)90229-5

44. Emerich, D. F., Thanos, C. G., Goddard, M., Skinner, S. J., Geany, M. S., Bell, W. J., et al. (2006). Extensive neuroprotection by choroid plexus transplants in excitotoxin lesioned monkeys. Neurobiology of Disease, 23(2), 471-480. https://doi.org/10.1016/ j.nbd.2006.04.014

45. Saporta, S., Cameron, D. F., Borlongan, C. V., \& Sanberg, P. R. (1997). Survival of rat and porcine Sertoli cell transplants in the rat striatum without cyclosporine-A immunosuppression. Experimental Neurology, 146(2), 299-304. https://doi.org/10. 1006/exnr.1997.6493

46. Acosta, S. A., Tajiri, N., Shinozuka, K., Ishikawa, H., Sanberg, P. R., Sanchez-Ramos, J., et al. (2014). Combination therapy of human umbilical cord blood cells and granulocyte colony stimulating factor reduces histopathological and motor impairments in an experimental model of chronic traumatic brain injury. PLoS One, 9(3), e90953. https://doi.org/10.1371/journal.pone.0090953

Publisher's Note Springer Nature remains neutral with regard to jurisdictional claims in published maps and institutional affiliations. 